\title{
DE INSTRUMENTOS MILITARES DE LA DICTADURA A INSTRUMENTOS POLÍTICOS DE LA DEMOCRACIA: REGÍMENES DE EXCEPCIÓN EN BOLIVIA 2000-2010
}

Claire Wright (elizabeth.wrightcl@uanl.edu.mx)

Universidad Autónoma de Nuevo León, México

Ana Soliz Landívar (ana.soliz@giga-hamburg.de)

German Institute of Global and Area Studies, Alemania

\begin{abstract}
El objetivo del artículo es descubrir el rol jugado por los regímenes de excepción en la política boliviana contemporánea. A pesar de constituir un legado clave de los regímenes autoritarios y sobrevivientes a las transiciones democráticas en América Latina, los Regímenes de Excepción han sido rara vez estudiados por los cientistas políticos. Centrándonos en el caso de Bolivia, la investigación se basa tanto en las leyes relevantes como en 65 decretos presidenciales, que son analizados mediante el método del análisis de contenido. Como resultado, se demuestra que para Bolivia, tanto el marco legal y el uso político de los Regímenes de Excepción se han transformado desde un mecanismo puramente represivo hacia uno más administrativo. El estudio contiene importantes implicancias sobre cómo los Regímenes de Excepción son conceptualizados desde un punto de vista teórico así como también para el entendimiento de la naturaleza de la política de emergencia de años recientes en democracias frágiles. Palabras clave: Bolivia, democracia, regímenes de excepción, presidentes.
\end{abstract}

\section{From Military InSTRUMENTS OF Dictatorship to POLITICAL INSTRUMENTS OF DEMOCRACY: REGIMES OF EXCEPTION IN BOLIVIa 2000-2010}

The aim of this study is to discover the role played by regimes of exception (RoEs) in contemporary Bolivian politics. RoEs have been seldom studied by political scientists, despite constituting a key legacy of authoritarian regimes that have survived the transitions to democracy in Latin America. Focusing on the case of Bolivia, the research is based on relevant laws as well as 65 presidential decrees, which are analyzed with the method of content analysis. As a result of the analysis, we show that in Bolivia both the legal framework and political use of RoEs have moved on from a purely repressive mechanism to a more administrative one. The study has important implications for how RoEs are conceptualized from a theoretical point of view as well as for our understanding of the nature of emergency politics in fragile democracies in recent years. Keywords: Bolivia, democracy, regimes of exception, presidents. 


\section{Introducción $^{1}$}

Los regímenes de excepción ( $\mathrm{RdE}$ ) pueden ser entendidos como un término que incluye aquellos mecanismos legales que, previamente designados, ofrecen poderes especiales en situaciones de emergencia. Comúnmente se les otorga diversos nombres en el nivel legal nacional -incluyendo "estado de sitio", "estado de emergencia", "estado de excepción”, “estado de alarma”, entre otros. En América Latina, los RdE se han transformado en sinónimo de dictaduras militares y de restricciones a los derechos civiles. Sin embargo, éstos han sobrevivido a la tercera ola de democratizaciones y todavía constituyen parte de la vida política Latinoamericana contemporánea. Salvo por el estudio pionero de Loveman, (1993) un análisis histórico, algunos pocos estudios de caso, ${ }^{2}$ y algunos análisis legales (entre otros, Carbonell, 2008; Delfino, 2000; García Sayán, 1987; Ríos, 2009; y Zovatto, 1990) ha existido poca preocupación académica motivada por el uso de estos mecanismos desde el comienzo de este período democrático ${ }^{3}$. Por su parte, la ciencia política ha ignorado el impacto potencial de estos mecanismos en la gobernabilidad democrática. Teniendo en mente esta aparente brecha en la literatura, ofrecemos entonces un estudio de caso sobre Bolivia, con el objetivo de clarificar cómo los RdE han sido utilizados durante la primera década del siglo veintiuno.

Bolivia es un caso interesante, puesto que su historia reciente ha seguido la tendencia general de la evolución de largo plazo en relación a los RdE en Latinoamérica, como fuesen identificados por Brian Loveman (1993) primero, la inclusión de mecanismos para suspender las garantías jurídicas, promulgada frente al descontento social durante el siglo diecinueve, segundo, un abuso de estos poderes por los gobiernos militares en el siglo veinte y, tercero, la permanencia del "estado de sitio" aun después de la transición a la democracia. No obstante, desde el 2000 en Bolivia un cambio legal y político clave ocurrió en relación a los RdE, pasando desde un

\footnotetext{
Las autoras quisieran agradecer a Mariana Llanos por su exhaustiva y constructiva revisión de una versión anterior de este texto; Salvador Martí i Puig por su dirección a través de la investigación; a Almut Schilling-Vacaflor, Otto Argueta, Salvador Alvídrez, Sara Mabel Villalba, y Aurelija Laurusonyte por su ayuda para establecer el sistema de codificación y llevar a cabo la codificación de las declaraciones presidenciales de los regímenes de excepción; y a todos aquellos entrevistados durante el curso de la investigación, particularmente a Horacio Andaluz por sus invaluables ideas. Una versión preliminar de este trabajo fue publicado en GIGA Working Papers N 218 (2013). Finalmente queremos agradecer el trabajo de los traductores y editores de Revista Política.

2 Sobre Colombia, ver Iturralde (2003) y Vázquez Carrizosa (1992).

3 Como ha enfatizado Zovatto (1990), los regímenes de excepción pueden y deben ser estudiados desde diversos puntos de vista: constitucional, político, económico y social.
} 
mecanismo que permitía la intervención militar en asuntos civiles a uno que ahora ofrece soluciones por la vía rápida tanto a desastres y emergencias naturales como humanos. Este cambio ha sido mucho más notorio en el caso de Bolivia que lo que ha sido por ejemplo en sus vecinos Ecuador o Perú (Wright, 2012).

En este estudio nos aproximamos al uso de los $\mathrm{RdE}$ en Bolivia tanto desde una perspectiva legal-normativa como desde una político-empírica, con el objetivo de analizar si estos mecanismos son todavía relevantes en la política del país y, si lo son, cuáles son sus principales características en el presente. Creemos que, teniendo en cuenta la falta de literatura y la relevancia de los RdE para la democracia, es necesario actualizar nuestro entendimiento de su rol actual en la política. En consecuencia, en este estudio ofrecemos un análisis empírico exhaustivo sobre un caso en particular, que puede servir de modelo para futura investigación sobre este mecanismo en otros países alrededor de América Latina.

Este artículo se encuentra dividido en cuatro secciones: primero, ofrecemos un marco teórico para los $\mathrm{RdE}$, prestando particular atención a la literatura sobre Latinoamérica; en segundo lugar, analizamos el marco normativo de los RdE en la legislación boliviana; en tercer lugar realizamos un análisis empírico de aquellos decretos presidenciales que hayan proclamado un $\mathrm{RdE}$ en este contexto, utilizando para ello análisis de contenido como metodología; finalmente ofrecemos algunas conclusiones y establecemos una agenda de investigación futura.

\section{Marco Teórico}

\subsection{Regímenes de Excepción en la Literatura}

Gross y Ni Aoláin (2006: 35) señalan que muchas democracias constitucionales (incluidas Francia, Alemania, y bastantes países en Latinoamérica) poseen un mecanismo pre-preparado en orden de lidiar rápida y eficazmente frente a una crisis $^{4}$. Estos mecanismos pueden ser establecidos en la constitución o en otro cuerpo de legislación ordinaria. De acuerdo a la teoría constitucionalista, este tipo de mecanismos es conocido como "régimen de excepción", que ofrece un marco normativo especial que puede ser puesto en marcha cuando una nación u orden constitucional se enfrenta a una "amenaza excepcional” (Friedrich, 1950).

La literatura no especifica cuál es la naturaleza de la crisis, algo que debe ser abordado en su propio mérito. 
El modelo clásico de un RdE es la institución de la dictadura en la Antigua Roma, la que se proclamaba en dos circunstancias principales:i) dictatura rei gerundae causa (con el fin de lograr hacer las cosas), que implicaba la necesidad de aplicar velozmente las decisiones políticas; $y$, ii) dictatura seditionis sedandae (para reprimir las insurrecciones), que se reduce al uso de la fuerza para detener las perturbaciones sociales (Rossiter, 1946: 20). La dictadura romana ha sido objeto de alabanza por variados pensadores a través de la historia, incluyendo a Friedrich (1950), Maquiavelo (edición de 1987), Rossiter (1946), y Rousseau (edición de 1990).La razón para esta admiración descansa en el hecho de que la dictadura contenía variadas limitaciones institucionales que ayudaban a evitar abusos de poder: primero que todo, una persona conocida y respetada (pero no un político activo) era elegido para administrar la dictadura, en orden a evitar la concentración de poder en manos de los Cónsules; segundo, un límite de tiempo de seis meses era impuesto, de manera de evitar que prevalecieran los poderes de emergencia de largo plazo; tercero, era el Senado el que calificaba una situación como de emergencia, mientras que eran los Cónsules quienes elegían al dictador; cuarto, no podía realizarse ningún cambio profundo a la República durante el reinado de una dictadura; y, quinto, este tipo de gobierno sólo podía ser proclamado una vez por año (Rossiter, 1946: 18-22).

De acuerdo a la teoría constitucionalista, los preceptos de la dictadura romana se transformaron en la base de los RdE modernos -vía la Edad Media, el Renacimiento, los Estados Absolutistas de Europa, y la Revolución Francesa- mediante el mecanismo del "estado de sitio", y finalmente en la Constitución de Weimar, mediante su Artículo 48 (Wright, 2012b). Los RdE modernos han preservado dos elementos claves de la dictadura romana: por una parte, la concentración de poder; y por otra, el establecimiento de un límite de tiempo (CruzVillalón, 1984; de Wilde, 2010). Más aún, han incorporado otro aspecto clave: la suspensión de los derechos humanos o las garantías jurídicas, algo que no había sido explícitamente considerado por la dictadura romana (Wright, 2012b).

Es importante destacar que la literatura actual sobre $\mathrm{RdE}$ se ha enfocado principalmente en la posibilidad de medidas represivas- tales como la suspensión de derechos humanos-, siendo usada frente a crisis sociales o políticas (Zovatto, 1990). Este énfasis en la dictatura seditionis sedandae ha involucrado que la dictadura rei gerundae, que era más un mecanismo administrativo que represivo, ha sido ampliamente obviada. Por ello, es importante recordar que los RdE tanto hoy como en Roma pueden ser más que meras expresiones de violencia gubernamental. En 
este estudio demostramos que las medidas administrativas pueden de hecho ser más comunes que las represivas, cuando los $\mathrm{RdE}$ son utilizados en la práctica.

\subsection{Regímenes de Excepción en América Latina}

El proceso de independencia de Latinoamérica vivido al comienzo del siglo diecinueve implicó una violenta lucha por el poder (Colomer, 1990:31; Loveman y Davies 1997: 3; Safford, 1987: 80-84). En este contexto, los Estados nacientes se enfrentaron al siguiente dilema: si estos deberían seguir las tradiciones autoritarias ibéricas, o en vez introducir el constitucionalismo liberal que estaba siendo defendido en esos tiempos por Francia, Gran Bretaña, y los Estados Unidos (Safford, 1987: 57). Como explica Roberto Gargarella, a pesar de que los conservadores y radicales tuvieron un impacto importante en el constitucionalismo de esta época, fue el activismo político de los liberales el que llevó a la adopción de las constituciones predominantemente liberales en la región (2005: 219).

Los principales preceptos del constitucionalismo liberal eran: proteger los derechos individuales; limitar el poder del Estado; restringir el poder presidencial; y, establecer un sistema de checks and balances (Gargarella, 2005: 197). Por esta razón, las primeras constituciones latinoamericanas no incluían poderes de emergencia, siguiendo en vez el "modelo de resistencia" (Gross y Ni Aoláin, 2006) que había sido propuesto por pensadores tales como Benjamin Constant (Aguilar Rivera, 1996: 27).

No obstante, sin que se hubiese creado respuesta constitucional para las situaciones de crisis enfrentadas por estos Estados nacientes (Loveman, 1993: 62), en muchas ocasiones sólo dos opciones estaban a su disposición: i) la remoción de los presidentes vía golpes de Estado [coup d'états] o ii) la violación de la constitución y los principios liberales, en orden de aferrarse al poder (Aguilar Rivera, 1996: 28). Consecuentemente, estos Estados liberalmente orientados eran minados por la deficiencia de su estructura institucional y, como explica Gargarella (2005: 247) los liberales Latinoamericanos habitualmente debían recurrir a principios más conservadores, incluyendo la concentración de poderes y el uso de la violencia en orden a obtener estabilidad. La consecuencia de esto fue que, al finalizar el siglo diecinueve, prácticamente todas las constituciones latinoamericanas habían incluido un marco para un RdE (Aguilar Rivera, 1996: 244; Loveman, 1993: 64; Safford, 1987: 62), sin que perdurase su renuencia inicial a hacerlo.

En esencia, el pensamiento liberal había demostrado ser inadecuado para la realidad política Latinoamericana. Por una parte, el modelo constitucional no ofrecía 
instrumentos que pudieran ser utilizados para afrontar las crisis que invariablemente emergieron durante la fundación de un nuevo Estado. Por la otra, la presencia de la "antipolítica" (Loveman y Davies, 1997) de los caudillos, en los sectores militares nacientes, y los mismos liberales, socavaron a estas jóvenes repúblicas. La respuesta sistemática a esta situación fue el uso de los RdE como mecanismo de represión oculta bajo un marco de legalidad mínima (Loveman, 1993: 203).

A pesar de que los militares cumplieron un importante rol durante la independencia y hasta finales del siglo diecinueve, con la formación de los primeros ejércitos en ese período, las fuerzas armadas en América Latina obtuvieron verdadera prominencia política luego de la Gran Depresión de 1929 (Rouquié, 1997: 289). Entre febrero y diciembre de 1930, los gobiernos de Argentina, Bolivia, Brasil, República Dominicana, Guatemala y Perú fueron todos derrocados por golpes de Estado militares. Esto se transformó en un evento común en Latinoamérica durante el siglo veinte, en muchos casos hasta finales de los 1980, incluso a pesar del breve período de "euforia democrática" que ocurre tras el fin de la Segunda Guerra Mundial (Rouquié, 1997). Los militares ganaron nuevamente creciente importancia luego de la Revolución Cubana de 1959, dadas las circunstancias de la Guerra Fría y el apoyo de los Estados Unidos en su lucha contra el comunismo. Entre 1962 y 1972 hubo una serie de golpes de Estado, que fueron seguidos por la represión violenta a los opositores de estos regímenes militares. Como explica Alain Rouquié (1997: 291), cualquier aspiración de cambio social o apoyo a partidos de izquierda era considerado como "revolucionario". Fue en este contexto que la doctrina de seguridad nacional fue desarrollada, con el orden público como la prioridad principal (Zovatto, 1990).

A pesar de que la ideología política de los militares ha mostrado cierto grado de variación -desde el histórico modelo autoritario burocrático en el cono sur a uno más progresivo en la región andina (Rouquié, 1997)-, los regímenes establecidos en América Latina durante el siglo veinte se caracterizaban finalmente por su común rechazo a la democratización (Loveman y Davies 1997: 222). Su solución a la amplia gama de problemas enfrentados era concentrar el poder, y en muchos casos limitar las libertades civiles en general, sea que el objetivo fuere la represión de opositores de la izquierda o el desarrollo económico de la nación. En orden de lograr una concentración de poder en el Ejecutivo, los militares recurrieron al uso de decretos, que buscaron legitimar mediante el uso de cláusulas de RdE que se encontraban presentes en las constituciones de los diferentes países (Loveman y Davies, 1997: 376). Efectivamente, para finales de los 1970 dos tercios de los países latinoamericanos vivían bajo "estados de excepción” (Despouy, 1999: 16). 
En términos reales, el uso y abuso de RdE era más común entre las dictaduras del cono sur. Como el mismo Despouy (1996) explica, las cláusulas de los RdE eran regularmente promulgadas en esta región, llevando al abuso perpetuo de los derechos humanos. De esta forma, los RdE adquirieron su forma moderna en América Latina bajo el gobierno autoritario del siglo veinte, sostenido por dos pilares fundamentales: la intervención de los militares en política y el uso de la fuerza para reprimir las revueltas sociales. Vale la pena señalar que la literatura sobre América Latina no cuenta con un registro claro de más $\mathrm{RdE}$ orientados a la administración aun cuando sospechamos que el modelo de dictadura rei gerundae ha estado presente en el pasado. En este sentido, es posible que la literatura haya obviado este importante aspecto de los RdE.

La llamada "tercera ola de democratizaciones" en América Latina comenzó al final de los 1970 -con la República Dominicana como la primera en transformarse, en 1978y culminó con el retorno a la democracia en Chile de 1990 (Rouquié, 1997:330). ${ }^{5}$ Las nuevas democracias enfrentaron dos desafios principales: por una parte, el legado de violencia institucionalizado y, por otra parte, las desigualdades sociales generalizadas en medio de la crisis económica de los 1980 (Kruijt y Krooning 2002: 24). Con respecto al primer desafio, como explica Terry Lynn Karl (1996), los militares llevaron a cabo una serie de pactos fundacionales con los nuevos actores democráticos en orden de mantener su poder e influencia en ciertas áreas. Consecuentemente, los nuevos gobiernos adoptaron una actitud pragmática, que en definitiva llevó a la impunidad y a un débil Estado de derecho (Loveman y Davies 1997: 367).

En la mayoría de los países latinoamericanos, la retirada de los militares del gobierno coincidió con nueva legislación electoral y nuevos arreglos constitucionales (Loveman y Davies, 1997: 226). Sin embargo Bolivia, Chile, y Uruguay eligieron mantener las constituciones existentes que habían sido diseñadas o usadas para sostener a los regímenes militares. Como ha sido mencionado, los pactos fundacionales fueron claves para que la supervivencia y éxito de las democracias emergentes, con la influencia de los militares en estas nacientes entidades reflejada en su condición de "democracias protegidas", que incluían elementos tales como códigos criminales que estipulaban cláusulas especiales para los crímenes políticos o que entregaban autonomía a los miembros de las fuerza armadas. Otro mecanismo que era muy importante para mantener la influencia de los militares fue precisamente los $\mathrm{RdE}$, establecidos como un mecanismo particularmente represivo. De hecho, cada una de las constituciones adoptadas entre 1978 y 1993 -con excepción de la paraguayareafirmaron y ampliaron las medidas que podían ser empleadas en una situación de emergencia (Loveman y Davies, 1997:376).

Es importante señalar que tanto El Salvador como Guatemala realizaron su transición a la democracia durante los 1990 . 
En el comienzo de esta tercera ola de democratización, hubo preocupación por parte de los académicos sobre el rol de los RdE y las posibles violaciones a los derechos involucradas. En 1986, la Comisión Andina de Juristas y el Instituto Interamericano de Derechos Humanos organizaron un seminario titulado "Los Estados de Emergencia en la Región Andina” (Figallo, 1987), que dio origen a una serie de textos que ofrecían estudios de caso desde dentro de la región andina (García Sayán, 1987). De la misma forma, Zovatto ofreció un análisis profundo sobre derechos humanos y estados de excepción en América Latina (1990). Para finalizar, en 1993 Loveman publicó su obra pionera y exhaustiva sobre regímenes de excepción en la historia de Latinoamérica, The Constitution of Tyranny.

Más de tres décadas después del inicio de la tercera ola de democratizaciones, los regímenes democráticos aún son débiles en la mayoría de los países latinoamericanos. Algunos autores consideran como falla fundamental de las democracias de la región, el uso arbitrario del poder por parte de las autoridades (Kruijt y Koonings, 2002: 44; O’Donnell, 1997: 183). Más aún, como resultado de la continua presencia de células terroristas, grupos paramilitares y traficantes de drogas en países como Colombia, México y Perú, la seguridad a cualquier costo es todavía un asunto clave; uno que los políticos ávidos de votos aprovechan de la mejor manera (Hagopian, 2005: 319).

Tomando esta situación en consideración, la tentación para los gobiernos de recurrir a los $\mathrm{RdE}$-que ofrecen una concentración del poder y la suspensión de las garantíasha sido enorme. Los miedos de los juristas tales como García Sayán (1987) y Zovatto (1990) desde el principio de esta tercera ola de democratizaciones parecen haber estado bien fundamentados, ya que este mecanismo ha sido habitualmente usado desde entonces hasta ahora en diversos países en Latinoamérica. De acuerdo a los reportes publicados por Naciones Unidas entre 1985 y 2005, ha habido por lo menos una declaración de estado de excepción en la gran mayoría de los países de la región (esto es, Argentina, Bolivia, Colombia, Ecuador, Guatemala, Honduras, Nicaragua, Paraguay, Perú y Venezuela). En el caso de Bolivia, la ONU ha registrado cuatro de estas declaraciones durante este período: en 1985, 1990, 1995 y $2000^{6}$. Si bien el reporte ofrecido por la ONU es ciertamente muy útil, vale la pena señalar que los datos sobre ciertos países son incompletos dadas las fuentes utilizadas: reportes en la prensa y reportes entregados por los gobiernos. Más aún, el tema ha abandonado la

\footnotetext{
6 Los informes anuales y las listas de Estados que, desde el 1 de enero 1985 han proclamado, prorrogado o levantado el Estado de Excepción fue presentada por Leandro Despouy, nombrado Relator Especial de acuerdo a la Resolución del Consejo Económico y Social 1985/37. Los informes entre 1993-2005 están disponibles en línea en: www.un.org
} 
agenda de la ONU desde 2005, sin que más reportes hayan sido publicados desde aquel año.

\section{El Marco Normativo de los Regímenes de Excepción en Bolivia}

La constitución boliviana de 1967 incluía el estado de sitio como un RdE. Como ya ha sido mencionado, este mecanismo se hizo famoso por ser usado en la práctica por los militares como un medio de represión. ${ }^{7}$ Las revueltas sociales y el estado de sitio declarados en abril del 2000, junto con mayor descontento social en septiembre del mismo año, hicieron necesario cambiar este marco legal en Bolivia. Con la ley $N^{\circ} 2140$ sobre "Prevención de riesgos y/o emergencias" de octubre del 2000, los RdE se hicieron más democráticos, removiendo la mención a las fuerzas armadas y constituyendo un recurso que, aun siendo excepcional, ya no incluye la restricción de los derechos humanos fundamentales. Este cambio fue reforzado por la nueva constitución del 2009. Sin embargo, la legislación sobre RdE sigue mostrando algunas contradicciones que serán destacadas a lo largo de este trabajo. (Horacio Andaluz, experto en constitucionalismo boliviano, afirma que: "La legislación boliviana en general, no ha sido coherente [...] la excepción a esta falta de coherencia es la jurisprudencia del Tribunal Constitucional, que siempre respetó sus propios precedentes o justificó por qué los había cambiado. Esto significaba que la jurisprudencia constitucional siempre fue sólida, no siempre es impecable, pero no contradictoria en sí misma Aquí me estoy refiriendo a la legislación y la jurisprudencia en general, es decir, no sólo el Estado de Sitio”. Entrevista realizada por Ana Soliz Landívar en Santa Cruz, Bolivia, enero 2012.)

\subsection{La Constitución Política del Estado de Bolivia de 1967}

A pesar de que la Constitución de 1967 fue promulgada por el gobierno democrático del General René Barrientos Ortuño, ésta continuó su vigencia durante un período de inestabilidad política y social, con frecuentes golpes de Estado. De hecho, el mismo General Barrientos había participado algunos años antes en el derrocamiento de Víctor Paz Estenssoro, en 1964. Más aún, Barrientos fue electo presidente en 1966 durante una elección donde fue vetado Víctor Paz Estenssoro, el único candidato aparte de Barrientos que tenía posibilidades reales de resultar electo (Gisbert y Gisbert, 2007: 512). Frente a este escenario de dudosa democracia

Entrevista personal con Ana María Romero llevada a cabo por Claire Wright en La Paz, Bolivia, abril de 2007. 
y el latente militarismo del presidente, la constitución fue diseñada, para otorgar un amplio poder en las fuerzas armadas las principales responsables de la mantención del orden interno del país. La Constitución de 1967 consideraba al estado de sitio como el único mecanismo constitucional mediante el cual se restauraba el orden en tiempos de lucha interna o guerra internacional. Fue creado esencialmente como un recurso de uso exclusivo del Ejecutivo.

De forma similar, se esperaba que luego de un máximo de 90 días el estado de sitio naturalmente expirara, con la excepción en casos de guerra civil o internacional, que permitirían su extensión. Sin embargo, esta excepción hacía posible la aplicación del estado de sitio perpetuo, dado que una de sus principales causas de ampliación eran precisamente la inestabilidad interna. De acuerdo a la constitución, no estaba permitida la declaración de más de un estado de sitio durante el mismo año a menos que el Congreso estuviera a favor de hacerlo; sin embargo, en muchas instancias el Congreso era disuelto por el gobierno de facto y como resultado su control no era implementado en la práctica.

El estado de sitio ofrecía medidas que podían tomarse "respecto de señaladas personas fundadamente sindicadas de tramar contra el orden público"8 e incluía citaciones judiciales y órdenes de arresto. En teoría, este mecanismo respetaba el habeas corpus, dado que la constitución establecía que la persona debía ser presentada ante el tribunal competente en menos de cuarenta y ocho horas desde el arresto. Adicionalmente, la proclamación de un estado de sitio permitía el cambio de la jurisdicción competente si las necesidades del orden público así lo requiriesen; por tanto, aquellos que fueren arrestados podían ser ubicados en cualquier lugar del territorio nacional. Por consiguiente, la "jurisdicción competente" podía ser en realidad cualquiera que el Ejecutivo designara como tal.

Una vez que el RdE acabase los ciudadanos serían liberados, excepto en aquellos casos en que un juicio hubiese comenzado en los tribunales competentes, los cuales -como ya hemos señalado- podían ser escogidos directamente por el Ejecutivo. El exilio político no estaba permitido y por lo mismo cualquiera que fuese confinado, buscado o arrestado por razones políticas no podía recibir el exilio, aunque formalmente, la constitución enfatizaba la garantía de ofrecer pasaporte a cualquier ciudadano que así lo requiriese. Con respecto a los efectos de la declaración de estado de sitio, estos

Constitución de 1967, artículo 112 párrafo 3, disponible en: http://www.vicepresidencia.gob.bo/ IMG/pdf/constitucion_1967.pdf 
eran vastos, yendo más allá de la restricción de los derechos antes mencionados. Por ejemplo, eran las fuerzas armadas las responsables de llevarlo a cabo.

Respecto de las responsabilidades en caso de cualquier abuso de poder de los servidores públicos civiles, es importante destacar que las reprimendas sólo eran impuestas sobre aquellos que estuvieran "siguiendo órdenes", y no respecto de aquellos que las daban. En la misma línea, es curioso notar que el mismo artículo en que se sancionaba a quienes violaran las garantías constitucionales, estaba estipulada la posibilidad de censura a la correspondencia y publicaciones en tiempos de guerra internacional. ${ }^{9}$

Las reformas de 1994, 2002, 2004 y 2005 mantuvieron el Artículo 111, con su regulación de la proclamación del estado de sitio. En esencia, no ha habido cambios mayores con respecto a la base establecida por la constitución de 1967. Más bien, el primer cambio sustantivo a las normas sobre los RdE en Bolivia ocurrió como resultado de la promulgación de la Ley N²140 el año 2000.

\subsection{Ley para la Reducción de Riesgos y Atención de Desastres No 2140 del 2000}

Esta ley fue promulgada el 25 de octubre del 2000 por el General Hugo Bánzer Suárez. Es importante señalar que tras esta ley estaba el conflicto de abril del 2000 -conocido en Bolivia como "La Guerra del Agua"- por la concesión de control sobre el suministro de agua a una compañía privada (de propiedad mayormente extranjera), que aplicó un aumento en las tarifas para el agua potable. Como resultado, un grupo ciudadano creó la llamada Coordinadora del Agua para demandar la inmediata cancelación del contrato de privatización del suministro de agua. Ante una escalada del conflicto, el Presidente Hugo Bánzer declaró un estado de sitio, que dejaría a varias personas muertas como resultado de la intervención militar. Al mismo tiempo que esto ocurría, miembros de la policía también se sumaron a las protestas demandado por aumento de sueldos. En el mismo año 2000, otra crisis gubernamental se desplegó cuando en septiembre cocaleros bloquearon la carretera central (La Paz-Cochabamba-Santa Cruz) por un período de tres meses (los cocaleros son los agricultores de la hoja de coca, que formaron un movimiento político liderado por Evo Morales, quien llegaría al poder en 2006)

Constitución de 1967, artículo 112 párrafo 5, disponible en: http://www.vicepresidencia.gob.bo/ IMG/pdf/constitucion_1967.pdf 
Por todo aquello, casi inmediatamente después de estos conflictos sociales, la ley 2140 se implementó en octubre del 2000 para establecer a los RdE como el mecanismo central para reducir los riesgos en casos de desastre y/o emergencia. Varios elementos clave son dignos de señalar. El primer artículo de la ley en cuestión incluye: primero, la idea de prevención; segundo, que los riesgos son vistos no sólo como sociales, sino también económicos; y, tercero, que las amenazas son ampliadas hasta incluir riesgos ocasionados por causas naturales, tecnológicas y/o humanas. Establece - de acuerdo al artículo 3- los principios para este nuevo tipo de $\mathrm{RdE}$, incluyendo: intereses y obligaciones colectivos; el derecho a la protección de la integridad física, infraestructura productiva, bienes y el medio ambiente; la responsabilidad de las instituciones públicas y privadas y también la de los individuos; planificación e inversión pública y privada para el desarrollo sustentable; y, el principio de la administración descentralizada, entregando a los gobiernos municipales la responsabilidad primordial de intervenir en caso de desastre o emergencia. Sin embargo, en la práctica estos gobiernos municipales, generalmente hablando, no poseen la capacidad suficiente para lidiar con este tipo de situaciones y por consiguiente necesitan contar con el apoyo de la prefectura del departamento y/o del gobierno central. Por ello, en realidad y a pesar de los principios de acción descentralizada y subsidiaria, la falta de recursos disponibles mantiene los $\mathrm{RdE}$ centrados en el nivel nacional. Uno de los desafios más grandes enfrentados cuando se declara un $\mathrm{RdE}$ es la coordinación entre las diversas instituciones nacionales y locales, como fue explicado por el funcionario de gobierno Manríquez, de la Subsecretaría para la Seguridad Ciudadana ${ }^{10}$.

La principal institución creada por la Ley No 2140 -como resultado del Artículo 6- es el Sistema Nacional para la Reducción de Riesgos y Atención de Desastres y/o Emergencias (SISRADE). Los objetivos del SISRADE son prevenir y reducir las pérdidas humanas, económicas, físicas, culturales y medioambientales causadas por desastres y/o emergencias. El SISRADE está compuesto por el Consejo Nacional para la Reducción de Riesgos y Atención de Desastres y/o Emergencias (CONARADE) como un órgano de decisión y coordinación superior, cuyo mandato es llevado a cabo por el Ministerio de Defensa Nacional y el Ministerio de Planificación y Desarrollo Sustentable. También está compuesto por instituciones y organizaciones de la sociedad civil a nivel nacional, departamental y municipal. El CONARADE es liderado por el Presidente de la República y compuesto por el Ministerio de Defensa Nacional - cuyo ministro puede actuar como la cabeza de

10 En una entrevista personal con Ana Soliz Landívar en la ciudad de La Paz, enero de 2012. 
la CONARADE en la ausencia del presidente- así como también el Ministerio de Finanzas, Ministerio de la Presidencia, Ministerio de Planificación y Desarrollo Sustentable, y el Ministerio de Gobierno.

\subsection{La Constitución de 2009 del Estado Plurinacional de Bolivia}

Cuando Evo Morales Aima asumió el poder en el 2006, Bolivia comenzó la promulgación de una serie de transformaciones institucionales, de las cuales la más importante fue la promulgación de la Constitución del Estado Plurinacional de Bolivia en el 2009. En ésta ya no se hace referencia a los estados de sitio, ya que la regulación de los $\mathrm{RdE}$ ha sido ampliada en general. Por tanto, la constitución ha establecido el principio de que en situaciones que representen peligros a la seguridad del Estado, amenazas externas, conflictos internos, o desastres naturales, el presidente del Estado tiene la capacidad de declarar un estado de excepción. Más aún, y bajo el supuesto de los que ya había sido establecido como resultado de la Ley $\mathrm{N}^{\circ} 2140$, la constitución del 2009 introdujo la seguridad del estado y confirmó a los desastres naturales como posibles pretextos para la declaración de un $\mathrm{RdE}^{11}$.

Otro cambio que fue introducido en la constitución del 2009 tenía que ver con la duración posible de un estado de excepción, que ahora depende de la aprobación previa de la Asamblea Legislativa Plurinacional. También corresponde a este estamento decidir las medidas a tomar durante un RdE. En esta forma, el estado de excepción no es más un instrumento exclusivo del Ejecutivo, ya que la Asamblea Legislativa ahora tiene un rol importante que jugar. Más aún, la constitución establece que los poderes excepcionales empleados en dichas circunstancias deben mantener una relación estricta y proporcional con cada suceso. La Tabla 1 resume las principales características de los distintos RdE establecidos dentro del marco normativo boliviano durante el período que va desde el 2000 al 2010.

\footnotetext{
11 Datos del constitucionalista Horacio Andaluz Vegacenteno obtenidos en una entrevista personal con Ana Soliz Landívar sostenida en Santa Cruz durante el mes de enero de 2012. En efecto, la promulgación de un $\mathrm{RdE}$ como resultado de un desastre natural era una cuestión de interpretación, ya que no se indica claramente en la definición de "Estado de sitio" incluido en la Constitución de 1967.
} 


\section{Tabla 1}

Características de los Regímenes de Excepción Incluidas en la Constitución o la Ley de Bolivia durante el período 2000-2010

\begin{tabular}{|c|c|c|c|}
\hline $\begin{array}{l}\text { Ley/ } \\
\text { Características }\end{array}$ & CPE 1967 & Ley 2140 de 2000 & CPE 2009 \\
\hline Nombre & "estado de sitio" & "desastre/emergencia" & “estado de excepción” \\
\hline Causas & $\begin{array}{l}\text { Conflicto interno, } \\
\text { Guerra internacional }\end{array}$ & $\begin{array}{l}\text { Prevención de y } \\
\text { protección contra: } \\
\text { Riesgos sociales y } \\
\text { económicos. } \\
\text { Amenazas de causas } \\
\text { materiales, tecnológicas } \\
\text { y/o humanas. }\end{array}$ & $\begin{array}{l}\text { Peligros a la seguridad } \\
\text { del Estado. } \\
\text { Amenazas externas. } \\
\text { Conflictos internos. } \\
\text { Desastres naturales. }\end{array}$ \\
\hline Autoridad & $\begin{array}{l}\text { El Presidente. } \\
\text { Más tarde, el Congreso } \\
\text { decide si continúa o no. }\end{array}$ & $\begin{array}{l}\text { Recomendación de } \\
\text { la CONARADE, } \\
\text { decretado por el } \\
\text { Presidente. }\end{array}$ & $\begin{array}{l}\text { Presidente de Bolivia } \\
\text { con aprobación } \\
\text { previa de la } \\
\text { Asamblea Legislativa } \\
\text { Plurinacional. } \\
\end{array}$ \\
\hline Instituciones & Presidencia & $\begin{array}{l}\text { Gobiernos Municipales } \\
\text { Prefecto del } \\
\text { departamento. } \\
\text { Gobierno nacional. } \\
\text { SISRADE. } \\
\text { CONARADE. }\end{array}$ & Presidencia. \\
\hline Período & $\begin{array}{l}90 \text { días, luego expira } \\
\text { automáticamente: } \\
\text { excepto en tiempos } \\
\text { de Guerra civil o } \\
\text { internacional. } \\
\text { No es posible declarar } \\
\text { más de uno un } \\
\text { mismo año. }\end{array}$ & $\begin{array}{l}\text { De acuerdo a la } \\
\text { necesidad. }\end{array}$ & $\begin{array}{l}\text { La Asamblea Legislativa } \\
\text { Plurinacional establece } \\
\text { la duración. }\end{array}$ \\
\hline Medidas & $\begin{array}{l}\text { Contra sospechosos, } \\
\text { arrestos, la } \\
\text { correspondencia } \\
\text { puede ser censurada, } \\
\text { garantía de inmunidad } \\
\text { parlamentaria. }\end{array}$ & $\begin{array}{l}\text { Principalmente } \\
\text { económicas y } \\
\text { administrativas. }\end{array}$ & $\begin{array}{l}\text { La Asamblea Legislativa } \\
\text { Plurinacional establece } \\
\text { las medidas a tomar. }\end{array}$ \\
\hline Militares & $\begin{array}{l}\text { Principal rol es hacer } \\
\text { cumplir el estado de sitio. }\end{array}$ & $\begin{array}{l}\text { Sin mención, sólo } \\
\text { se refiere al Servicio } \\
\text { Nacional de Defensa } \\
\text { Civil SENADECI. }\end{array}$ & Sin mención. \\
\hline Control & $\begin{array}{l}\text { Sanciones sólo en contra } \\
\text { de aquellos que "siguen } \\
\text { órdenes". }\end{array}$ & $\begin{array}{l}\text { Responsabilidades } \\
\text { de acuerdo a las leyes } \\
\text { generales respectivas. }\end{array}$ & $\begin{array}{l}\text { Ejecutivo responsable } \\
\text { ante la Asamblea } \\
\text { Legislativa Plurinacional. }\end{array}$ \\
\hline
\end{tabular}

Fuente: Compilación de los autores. 


\section{Una Aproximación Empírica a los Regímenes de Excepción en la Política Boliviana Durante el Período 2000-2010 ${ }^{12}$}

A modo de entender cómo estos RdE han sido promulgados en Bolivia, buscamos aquellos decretos presidenciales que se refieren a estos mecanismos de forma explícita o implícita durante un período de once años: 2000-2010. Es importante destacar que por explícita entendemos aquellos decretos que incluyen una referencia al $\mathrm{RdE}$ como ha sido establecido en la constitución o las leyes; por implícito, queremos decir aquellos decretos que emplearon la nomenclatura de un RdE como está establecida por la constitución o las leyes, y que siguen el formato de los decretos de RdE explícitos, pero que no se refieren directamente al mecanismo legal ${ }^{13}$. Se hallaron un total de 65 decretos, demostrando que los $\mathrm{RdE}$ han sido de profunda importancia en la vida política boliviana reciente. Sin embargo, en orden a establecer el propósito que se ha atribuido a estos mecanismos, es necesario entender su contenido. Por esta razón, elaboramos un sistema de variables y categorías mutuamente excluyentes luego se asignaron valores a cada una de estas variables para cada decreto. De esta manera, fue posible convertir el texto de los decretos a una base de datos relevante con información para este estudio.

\subsection{Análisis de contenido}

Para este estudio realizamos análisis de contenido, que ofrece un enfoque inductivo que sin embargo respeta los principios claves del método científico -incluyendo confiabilidad, validez, replicabilidad y generalización- (Neuendorf, 2002: 50-51; Buttolph, Johnson y Joslyn, 1995: 245-253). La confiabilidad ha sido lograda por dos codificadores que revisaron para asegurar que el sistema de codificación produjese los mismos resultados en pruebas distintas. De la misma manera, la validez se alcanzó a través de las diferentes variables y categorías que se obtuvieron como resultado de un proceso de ensayo y error, que entonces aseguró que estábamos midiendo aquello que en realidad queríamos medir. Más aún, el estudio es replicable porque las variables y categorías son lo suficientemente amplias para ser usadas en países distintos. Finalmente, busca ser generalizable dado que la población completa de decretos relevantes fue estudiada y por lo mismo no hay preocupación por sobredimensionar la muestra (Neuendorf, 2002: 12).

12 El contenido de esta sección es una adaptación de Wright (2012).

13 Creemos importante incluir todos estos casos, dado que los decretos son actos presidenciales de gobierno que pueden no ser de "legalidad floja" -en el sentido que puede haber una supervisión inadvertida u omisiones de información del servidor público a cargo de elaborar el decreto. 
Creemos que el análisis de contenido es una herramienta poderosa para abordar nuestro cuerpo de datos: 65 decretos presidenciales que en esencia son textos. Como explican Buttolph, Johnson y Joslyn (1995: 252), el análisis de contenido ofrece un medio de abordar temas complejos y grupos de textos a través del tiempo. Por esta razón, lo elegimos como la principal herramienta a través de la cual analizamos los decretos considerados en este estudio.

Las variables e indicadores identificados como base de este trabajo se encuentran organizadas de acuerdo a cuatro dimensiones clave: primero, los elementos de identificación general; segundo, elementos formales; tercero, las situaciones referidas como emergencias; y, cuarto, las medidas empleadas. En orden a encontrar variables adecuadas e indicadores, llevamos a cabo una inmersión cualitativa en los decretos. En esta etapa, contamos con el apoyo de varios investigadores y llevamos a cabo un diálogo entre texto y teoría, de acuerdo con las recomendaciones de la grounded theory (para mayor información sobre dicha teoría ver Glasser y Strauss, 1967). A continuación, identificamos los hallazgos principales y las diferentes variables establecidas para cada dimensión como resultado de esta inmersión cualitativa.

\section{Dimensión 1: Elementos de Identificación Generales}

A modo de vincular los decretos con su contexto, parecía importante establecer algunas variables sencillas, incluyendo:

a) el número de referencia del decreto; la fecha en que fue firmado; la fecha en que fue publicado;

b) el presidente que firmó el decreto;

c) la nomenclatura usado en la declaración ("estado de sitio", "desastre", “emergencia”, "estado de excepción”).

\section{Dimensión 2: Elementos Formales}

Como se señaló en el delineamiento de nuestro marco teórico, la literatura sobre los poderes de emergencia ha mostrado una tendencia a centrarse en los elementos formales en vez del contenido material de estas medidas. Como señalan As Gross y Ni Aoláin (2006), hay varias formas de clasificar las respuestas a emergencias desde una perspectiva más procesal. Además, en el caso de los modelos constitucionales de acomodación -o RdE- los estudios se han centrado en cuestiones tales como los plazos, la supervisión y el área de su aplicación. Por lo tanto, decidimos tomar en cuenta estas variables en el análisis con la finalidad de ofrecer una pequeña contribución a la discusión existente en la literatura. Sin embargo, como ya estará 
claro, el objetivo de este estudio se centra tanto en los elementos materiales como los formales. Como resultado de la inmersión cualitativa y el diálogo con la teoría existente sobre $\mathrm{RdE}$, las siguientes cinco variables fueron creadas para esta dimensión:

a) $\mathrm{RdE}$ - variable nominal con tres valores: constitucional, legal, o sin referencia explícita;

b) otras leyes - una variable nominal, dicotómica, con dos valores: sí (si existe referencia a otras leyes) o no (si no hay referencia a otras leyes).

c) Límite temporal - una variable nominal, dicotómica, con dos valores: sí (si existe un límite de tiempo explícito) o no (si no existe límite temporal explícito).

d) Área de aplicación - una variable nominal con siete valores: territorio nacional; territorio sub-nacional; sector o servicio a nivel nacional; sector o servicio a nivel sub-nacional; grupo social; proceso político; o, no puede ser determinando.

e) Supervigilancia - una variable nominal con ocho valores: Congreso; el Tribunal Constitucional; el Ejecutivo; el órgano encargado del manejo de desastres; autoridad subnacional; organizaciones civiles; combinación de las anteriores; o, ninguna.

\section{Dimensión 3: Situaciones}

La tercera dimensión se relaciona con las situaciones en cuyo nombre las medidas fueron decretadas. En la literatura clásica sobre RdE hay cuatro principales circunstancias: la guerra (civil o internacional); desastres naturales; amenaza para el orden interno; o bien, una crisis económica. De acuerdo a la ley constitucional de Bolivia, cualquiera de ellos podría representar una "amenaza excepcional" a la vida de una nación y su forma normal de funcionamiento. Sin lugar a dudas, en la inmersión cualitativa hubo referencias a los desastres naturales, las amenazas para el orden interno y los problemas socioeconómicos, pero en realidad la situación era más compleja que causalidades singulares, y como tal, vale la pena señalar varios puntos.

En primer lugar, cabe destacar que hemos encontrado una tendencia a referirse a una mezcla de diferentes circunstancias dentro de una misma situación de emergencia. Por lo tanto, es necesario reconocer que estas circunstancias no son mutuamente excluyentes, y debemos pensar en la "amenaza excepcional" de una manera diferente. Por ejemplo, es posible encontrar referencias a una situación de protesta y desastres naturales en un mismo decreto. De la misma manera, es necesario señalar que a pesar de que las emergencias son entendidas por la literatura como 
de naturaleza "socioeconómica", descubrimos que las dificultades se encuentran en la administración o gestión de ciertos sectores o servicios en vez de en la macroeconomía o las situaciones sociales como tales. Más aún, en dichos casos en que se trata de un problema social como el desempleo, la dificultad es en realidad la presencia de tensiones sociales o conflictos potenciales más que los problemas económicos per se. Por tanto, en este análisis hemos reconstruido la clasificación de emergencias como socioeconómicas para hablar por una parte de problemas administrativos y de las tensiones sociales por la otra. Desde esta perspectiva más matizada, es posible establecer diferentes variables y ofrecer algunos indicadores que provienen directamente de los decretos mismos:

a) Desorden interno - protestas, enfrentamientos violentos, coups d'état, subversión, abandono de las tareas por parte de la policía.

b) Inseguridad - tráfico de drogas, lavado de dinero, robos, tráfico de personas;

c) Tensiones sociales latentes - pobreza, desempleo, condiciones de vida infrahumanas ${ }^{14}$;

d) Mala gestión de un sector o servicio - corrupción, especulación, actividades económicas informales, condición precaria de la infraestructura, contrabando; e) Interrupción en las actividades de un sector o servicio - escasez de gas, calles bloqueadas, bajos niveles de producción en el sector agrícola;

f) Plan o procedimiento que todavía debe ser implementado en el sector o servicio: reforma agraria, plan de desempleo, plan de trasladar un sitio de descarga desde una ubicación a otra;

g) Fenómeno natural adverso - sequía, inundaciones, erupciones volcánicas, incendios forestales;

h) Polución ambiental - cualquier cosa relacionada a polución. Problemas de sanidad - influenza, dengue, heridas, falta de disponibilidad de agua para consumo humano ${ }^{15}$.

\section{Dimensión 4: Medidas}

La cuarta dimensión está relacionada con las medidas adoptadas vía decretos. La literatura constitucionalista se refiere a la suspensión de los derechos humanos y la concentración

14 En algunos decretos, se encontraron referencias a "conmoción interior" (lucha interna), que en algunos casos podría ser similar a la tensión social. Fundamentalmente, el concepto carece de un significado específico y por lo tanto parece ser más un elemento que justifica que una situación en sí mismo.

15 Consideramos que el extenso brote de la enfermedad en el ganado sea una situación de emergencia, teniendo en cuenta su impacto en el sector agrícola (y por lo tanto, no consideramos que sea una parte del escenario de problemas de salud). 
de los poderes en la rama ejecutiva de gobierno, pero poco más. Para esta parte, la literatura sobre los poderes de emergencia legislativos ha establecido que todas las leyes promulgadas tienden a ser de naturaleza económica. Sin embargo, en los decretos aquí estudiados encontramos en realidad situaciones distintas. Por lo tanto establecimos las siguientes variables dentro de esta dimensión, junto a algunos indicadores:

a) Suspensión de derechos humanos: suspensión de artículos de las diferentes constituciones relacionados con derechos humanos, censura a los medios, prohibición de la libertad de movimiento;

b) Uso de los militares: nombramiento de las fuerzas armadas para vigilar caminos e instalaciones, militarización de la población, movilización militar, nombramiento de las fuerzas armadas como garantes del orden público;

c) Uso de la fuerza: confiscación de bienes o servicios, control de las áreas fronterizas, mantenimiento del orden público en general;

d) Intervención en la gestión de un sector:la idea es intervenir en el funcionamiento o estado de un sector o servicio con medidas de tipo administrativo, por ejemplo mediante el cambio de director, reemplazo de archivos administrativos, creación de empleo, ofrecer crédito o entrega de subsidios;

e) Exoneración de procedimientos administrativos o presupuestarios ordinarios: no tener que abiertamente ofertar para obtener contratos públicos, desviar fondos destinados a otras áreas para cubrir los costos de la emergencia;

f) Rehabilitación de un servicio o sector: la idea es regresar a su estado original construcciones, sectores o infraestructura vía trabajos o compras, por ejemplo mediante la reconstrucción de un puente, reparación de caminos, rehabilitación del sector agrícola;

g) Ayuda humanitaria: la idea es ofrecer primeros auxilios a personas afectadas por una situación que afecte seriamente su forma de vida bajo el modelo de manejo de riesgos, por ejemplo entregando a los refugiados camas y comida, cooperación para aquellos afectados, ayuda inmediata;

h) Atención médica: prevención de epidemias, asistencia médica;

g) Medidas técnicas: la idea es ofrecer una respuesta inicial en una situación de riesgo o daño material o al medioambiente bajo el modelo de manejo de riesgo, por ejemplo controlando un incendio forestal, llevando a cabo actividades para reducir riesgos pero no reconstruyendo, implementando acciones y proyectos de emergencia, haciendo referencia a una intervención por parte de la CONARADE. 


\subsection{Resultados del Análisis de Contenido de las Declaraciones de Regímenes de Excepción}

Con respecto a la formación en el proceso de codificación, contamos con la ayuda de dos investigadores en Ciencia Política de la Universidad de Salamanca, quienes codificaron el 10 por ciento de las unidades de análisis (7 decretos presidenciales). la fiabilidad global -medida en términos del porcentaje de acuerdos entre dos codificadores- fue del 87 por ciento, un nivel mucho más alto que la brecha usual para este tipo de estudio, del 70 por ciento. Al nivel de las variables individuales, todas ellas presentaron un porcentaje de acuerdo del 70 por ciento, con excepción de dos casos: la variable "otras leyes", con un 57 por ciento, y la variable "supervisión", con sólo un 14 por ciento de acuerdo. Hay varias razones distintas que pueden explicar por qué estas variables no pudieron ser codificadas satisfactoriamente, ${ }^{16}$ pero en todo caso fueron eliminadas del estudio para que no afectaran el porcentaje global de acuerdo. A continuación presentamos los resultados del análisis de contenido para las cuatro dimensiones establecidas previamente.

\section{Dimensión 1: Elementos de Identificación Generales}

Como ya ha sido destacado, descubrimos 65 decretos que declaran $\mathrm{RdE}^{17}$, promulgados por los diferentes presidentes bolivianos en el período entre el 2000 y 2010. Con respecto a las nomenclaturas, 2 casos corresponden a "estados de sitio", en 52 casos a una "emergencia" y en 11 casos se declaró un "desastre". Vale la pena señalar que no hay declaraciones de "estado de excepción" durante el período estudiado aquí.

Luego fue de interés hallar cuántos decretos fueron promulgados por año en el período estudiado para comprobar si hay una tendencia estable a través del tiempo o si existió concentración de decretos en un período o año en particular. En este sentido, es curioso señalar que hubo un incremento en el número de decretos durante el 2005, justo antes que Evo Morales se transformara en presidente y cuando el modelo

16 En el caso de la variable sobre otra legislación una buena explicación sería que los investigadores son científicos políticos en lugar de expertos legales, mientras que en el caso de la variable de control podría ser debido a la falta de claridad en los decretos sobre la posibilidad de la supervisión de la RdE.

17 Se realizó una búsqueda en la Gaceta Oficial del Estado boliviano con las mismas palabras y los términos empleados en las nuevas establecidos en la Constitución de 1967 ("Estado de sitio"), en la Ley N 2140 de 2000 ("emergencia” o "desastre ") y en la constitución de 2009 ("Estado de Excepción"). Hemos encontrado 53 decretos con la nomenclatura "emergencia", diez con “desastre" y dos con "Estado de Sitio". Ninguno de los decretos declaró "Estado de Excepción”, la nomenclatura de la Constitución de 2009. 
de "democracia pactada" se encontraba en crisis (para una descripción detallada y un análisis de la "democracia pactada" en el caso boliviano, consulte el volumen colectivo editado por Domingo, 2006). Después, durante el gobierno de Evo Morales (2006-2010), observamos una tendencia decreciente en la declaración de RdE. El gráfico a continuación (Figura 1) presenta estas fluctuaciones.

Figura 1

Declaraciones Presidenciales de Regímenes de Excepción por Año en el Caso de Bolivia, 2000-2010.

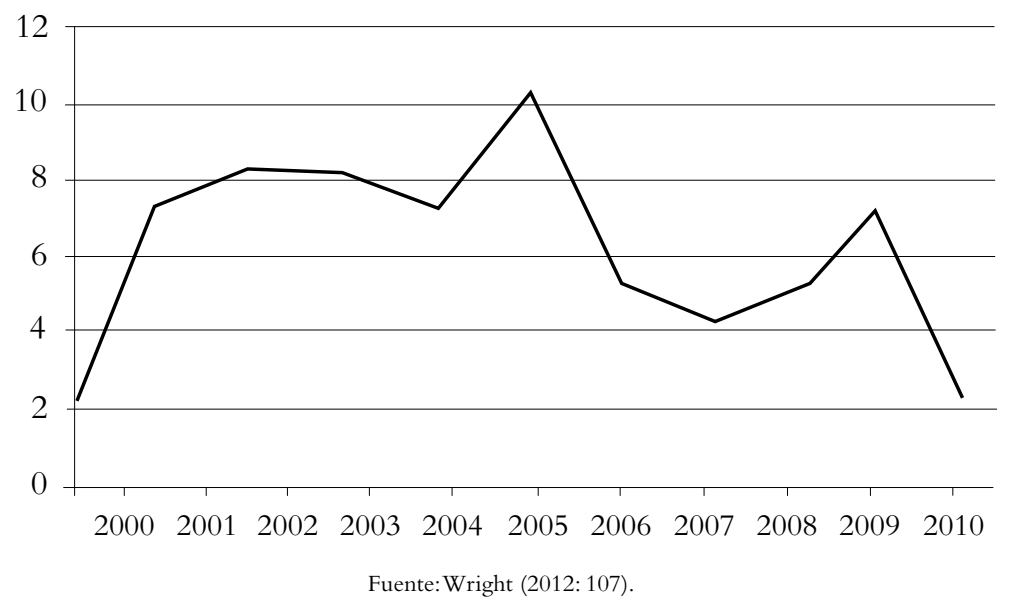

Similarmente, observamos el número de decretos de $\mathrm{RdE}$ promulgados por cada presidente porque representan una importante demostración de autoridad ejecutiva. A pesar de que Evo Morales es el presidente más “excepcional” en términos netos del período estudiado (con 22 declaraciones de este tipo), vale la pena recordar que durante este espacio de tiempo él estuvo en la presidencia por cinco años. Para comparar a los diferentes presidentes de manera igualitaria, entonces, fue calculado un promedio del número de decretos promulgados por períodos de seis meses ${ }^{18}$, un marco de tiempo que corresponde a la administración presidencial más breve de este período, aquella de Eduardo Rodríguez Veltzé. De acuerdo a estos

18 Los presidentes que ejercieron el cargo durante el período 2000-2010 fueron: Hugo Bánzer (hasta agosto del 2001), Jorge Quiroga (agosto 2001-agosto 2002), Gonzalo Sánchez de Lozada (agosto 2002-octubre 2003), Carlos Mesa (octubre 2003-junio 2005), Eduardo Rodríguez Veltzé (junio 2005-enero 2006) y Evo Morales (enero 2006 al presente). 
criterios, Rodríguez Veltzé mostró la mayor tendencia a recurrir a los RdE durante su posesión del cargo, con un promedio de seis decretos promulgados por período de seis meses en el gobierno. Esto es un contraste crudo con Hugo Bánzer - un exdictador- que tuvo el promedio más bajo (apenas sobre uno). La Figura 2 grafica estos resultados.

Figura 2

Número Promedio de Declaraciones de Regímenes de Excepción por cada Presidente Boliviano, Calculado por Períodos de Seis Meses entre 2000-2010

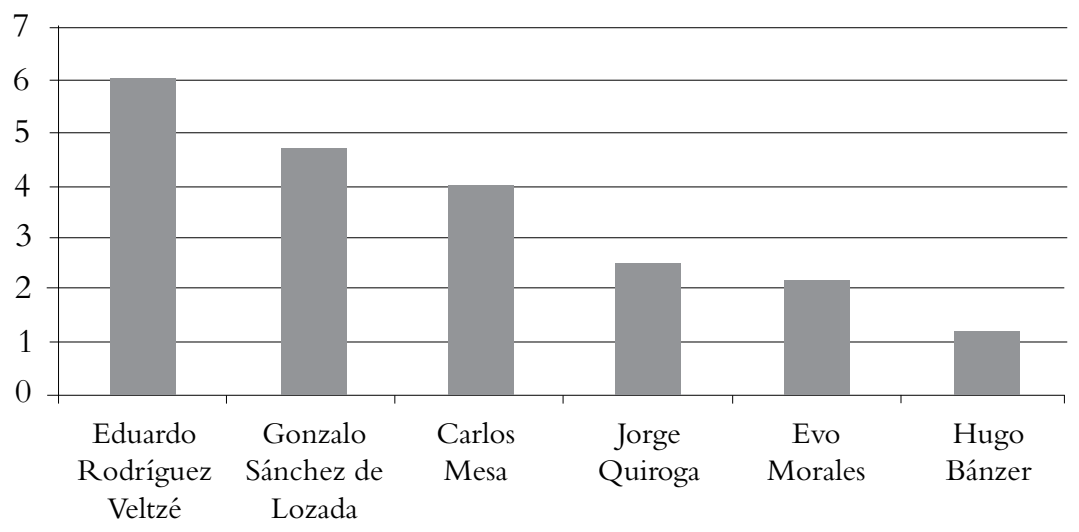

Fuente: Adaptación de Wright (2012).

\section{Dimensión 2: Elementos Formales}

Como ya ha sido mencionado, la literatura se ha centrado exclusivamente en los elementos formales de los poderes de emergencia en general y de los $\mathrm{RdE}$ en particular, obviando entonces más los elementos materiales. La contribución de este estudio es explorar ambos aspectos. Sentimos que es de interés reportar nuestros descubrimientos relacionados con los elementos formales para revelar el grado en que el uso de RdE en Bolivia refleja lo que pensamos que podemos comprobar teóricamente acerca de ellos. Vale la pena recordar que en la prueba de fiabilidad intercodificadores, dos de las variables para esta dimensión tenían muy bajos niveles de acuerdo y por tanto debieron ser excluidas del análisis final.

Con respecto al marco legal, la declaración de un $\mathrm{RdE}$ estaba basado principalmente en la Ley $\mathrm{N}^{\circ}$ 2140, con 49 decretos. La constitución de 1967 fue la base legal explícita para la declaración de dos decretos. Al mismo tiempo, fueron hallados 14 
decretos en que una "emergencia" o "desastre" fue declarado (la nomenclatura del RdE incluida en la Ley $\left.N^{\circ} 2140\right)$, una situación identificada como excepcional, estableciéndose medidas extraordinarias en respuesta. Sin embargo, en estos decretos no hay referencia explícita a un RdE incluido en la legislación boliviana. Estos decretos podían ser calificados como "paralegales", dado que emplean la misma terminología que aparece en el marco legal de los RdE y siguen el mismo formato que la declaración explícita, pero adolecen precisamente de referencia explícita a la base legal que es parte del concepto de RdE mismo. De acuerdo a nuestros criterios de investigación, es importante incluirlos en el análisis dada la naturaleza legal y política del análisis empírico (en este sentido seguimos, en vez de desafiar, el precedente establecido por Rubio y Goretti (1998) en su análisis de los decretos de necesidad y urgencia en el caso Argentina).

Con respecto al límite de tiempo, llama la atención que la gran mayoría de los decretos no estipula la duración de la declaración. En sólo 14 decretos había una referencia a un límite de tiempo específico o general. Por un lado, esto puede deberse al hecho de que, en el marco de la Ley $N^{\circ} 2140$, todo decreto de este tipo debía luego ser levantado por un decreto separado. ${ }^{19}$ Sin embargo, la falta de reconocimiento explícito de que la aplicación de estos decretos era temporal, ciertamente nos hace cuestionarnos la conceptualización tradicional de los $\mathrm{RdE}$.

Finalmente, es interesante tomar en cuenta el área de aplicación de las declaraciones. El territorio subnacional es el área más comúnmente referida (con 41 casos), seguida por el territorio nacional (14 casos), un sector al nivel nacional (4 casos), un sector al nivel subnacional (2 casos), un grupo social (1 caso) y un proceso político (1 caso). En dos casos no es claro a cuál área se aplica la declaración, lo que es curioso en sí mismo. En todo caso, es posible concluir que la vasta mayoría de declaraciones se aplica a un territorio específico, sea este nacional o subnacional. Sin embargo, es también necesario señalar que diez casos representan una innovación aparente, dado que se aplican a sectores o servicios económicos, grupos sociales, procesos políticos e incluso a áreas no identificables.

Con esta breve presentación de los resultados de nuestra segunda dimensión de estudio, hemos intentado colaborar con la literatura sobre RdE existente. Hemos hecho esto para demostrar que en la práctica ciertos elementos no son seguidos o adheridos siempre, y como tales puede por tanto no ser necesario revisar la teoría de tipo de procedimental sobre estos mecanismos.

19 Entrevista con el oficial de gobierno Lic. Manríquez, La Paz, enero de 2012. 


\section{Dimensión 3: Situaciones}

La tercera dimensión se refiere a las circunstancias definidas como "excepcionales", en nombre de las cuales se toman las medidas extraordinarias. Vale la pena reiterar que descubrimos referencias a una mixtura de diferentes situaciones dentro de un mismo decreto. Los resultados del análisis que se presentan en esta sección se encuentran en términos brutos, y reflejan el número total de referencias hechas a las diferentes situaciones en el corpus de 65 decretos escrutados.

\section{Figura 3}

Situaciones a las que se hace Referencia en los Decretos Proclamando Regímenes de Excepción en Bolivia entre 2000-2010

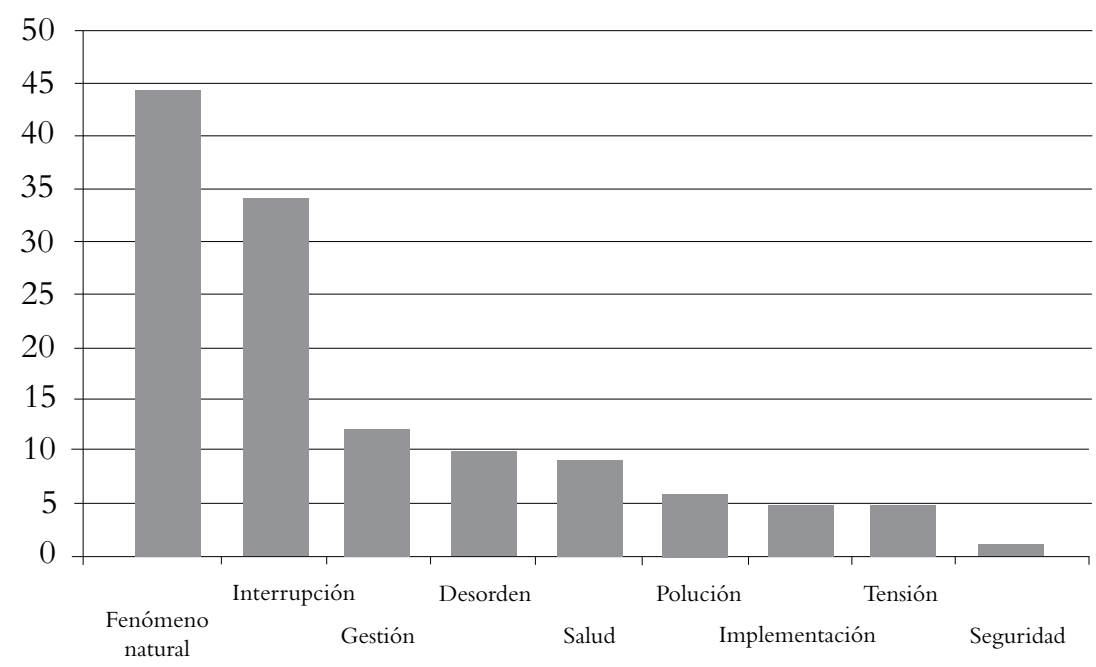

Fuente: Adaptación de Wright (2012: 110).

Uno de los resultados más impactantes es la presencia considerable de fenómenos naturales adversos (44 casos), al igual que la interrupción de actividades económicas o servicios públicos (34 casos). Más aún, los niveles relativamente bajos de desorden público (10 casos), desafian la conceptualización clásica de los RdE en América Latina, que postulan a la lucha interna como la causa clave de este tipo de decretos. Finalmente, llama la atención que sólo en un caso se hace referencia a problemas de inseguridad como motivo para la declaración de un RdE. 


\section{Dimensión 4: Medidas}

Habiendo presentado los resultados sobre las varias situaciones a las que se hace referencia en estos decretos, es ahora lógico enfocarse en las medidas empleadas en su nombre.Vale la pena señalar que hubo aquí una tendencia similar a presentar una mezcla de medidas diferentes dentro del mismo decreto. Los resultados del análisis son presentados en esta sección como términos brutos, y reflejan el número total de referencias hechas sobre las diferentes medidas en el corpus de 65 decretos escrutados.

Figura 4

Las Medidas Establecidas en los Decretos que Proclaman Regímenes de Excepción en Bolivia entre 2000-2010

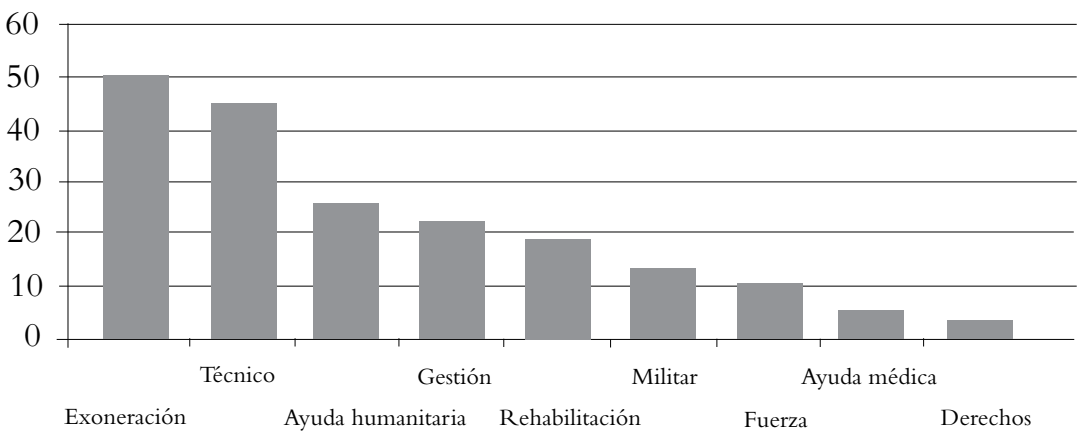

Fuente: Adaptación de Wright (2012: 112).

Los resultados de este análisis exploratorio de las medidas empleadas vía estos decretos son también muy reveladores. Por una parte, observamos una tendencia considerable a permitir excepciones de los procedimientos normales de licitaciones públicas y procedimientos presupuestarios, así como también a dar mayor asistencia técnica en el contexto de la prevención de riesgos. Por otra parte, los derechos humanos solo fueron suspendidos en dos casos, hecho que refleja la marcada tendencia a evitar la declaración de mecanismos de "estado de sitio", lo que desafia la conceptualización clásica de $\mathrm{RdE}$ como sinónimo de suspensión de los derechos humanos (particularmente cuando se emplean en la práctica). No obstante lo anterior, en 12 casos las fuerzas armadas fueron llamadas, lo que en sí mismo constituye una amenaza potencial a la gobernabilidad democrática. 


\section{Conclusión}

El objetivo de este trabajo ha sido analizar el rol de los regímenes de excepción ( $\mathrm{RdE}$ ) en la política boliviana entre el 2000 y 2010. Esto ha sido investigado vía análisis empírico, tanto en las normas legales como de los decretos presidenciales. Creemos que el trabajo es particularmente relevante dada la falta de estudios sistemáticos realizados hasta ahora sobre este tipo de mecanismos en América Latina, a pesar de la amplia utilización de los mismos por gobiernos autoritarios y democráticos en la región.

Por otra parte, descubrimos que el marco normativo que sustenta los RdE pasó por una serie de importantes cambios. El mecanismo inicial fue el estado de sitio, establecido por la Constitución de 1967, que facilitaba la intervención militar y la suspensión de los derechos humanos para reprimir los conflictos internos. En el 2000, con la Ley $\mathrm{N}^{\circ} 2140$ para la prevención de riesgos y el alivio de desastre y las figuras de "desastre" y "emergencia", los RdE en Bolivia se convirtieron en un mecanismo mucho más democrático, primero porque el rol de los militares fue reducido al manejo de desastres y, segundo porque las causales fueron ampliadas incluyendo desastres naturales y económicos. Finalmente, la nueva Constitución de 2009, con su "estado de excepción" parece combinar elementos de ambos mecanismos: por una parte, enfatiza la necesidad de respetar los derechos humanos y amplía las causales incluyendo desastres naturales, mientras que por otra parte mantiene vivos los temas tradicionales cómo la seguridad del Estado y las amenazas tanto internas como externas. Indudablemente, estos desarrollos legales son altamente significativos para Bolivia.

Habiendo llevado a cabo un análisis legal, procedemos a conducir una investigación empírica de las diferentes declaraciones de RdE entre el 2000 y 2010. En términos generales, podemos señalar que durante este período los presidentes bolivianos promulgaron un total de 65 decretos que proclamaban RdE. La Ley $N^{\circ} 2140$ entrega el marco legal para la vasta mayoría de estos decretos, con sólo dos referencias hechas a los RdE incluidas en la constitución de 1967. Más aún, es interesante observar que hasta el final de 2010 ningún decreto ha sido promulgado con referencia al "estado de excepción" que fuese establecido por la constitución de 2009, a pesar del hecho de que el marco legislativo en ésta es mucho más amplio de lo que es en relación al "estado de sitio" de las Constitución de 1967. De la misma forma, es necesario llamar la atención sobre los 14 casos en que no hubo referencia explícita a 
la constitución o a la Ley $N^{\circ} 2140$, que meramente siguieron el formato y lenguaje de otros decretos.

En términos de elementos materiales en estos decretos, nuestro análisis sugiere que su propósito principal no era actuar como un medio para que los militares pudiesen reprimir los desórdenes sociales, como la literatura tradicionalmente indica. En términos de las situaciones a las que se hace referencia en estos decretos, las más frecuentes estaban relacionadas a desastres naturales y a la disrupción de actividades económicas y servicios públicos. Finalmente, con respecto a las medidas empleadas, es bastante llamativo el alto número de incidencias de exoneración de los procedimientos presupuestarios de licitación y contrato público al igual que el incremento del uso de ayudas técnicas en el contexto del manejo de riesgo.

Como resultado de nuestro análisis, hemos sido capaces de demostrar cómo los $\mathrm{RdE}$ en Bolivia han evolucionado de ser instrumentos militares de dictadura a instrumentos políticos en democracia, ahora usados predominantemente como forma de lidiar con los riesgos que representan los desastres naturales. Mientras que esto representa indudablemente una buena noticia para la consolidación democrática en el país, también genera una pregunta nueva e importante, que es de enorme importancia en el contexto de la gobernabilidad democrática: ¿qué impacto tiene la exoneración de los procedimientos presupuestarios de licitación y contrato público en el uso y transparencia de los recursos públicos en Bolivia? Sin embargo, la respuesta a esta crucial pregunta se encuentra fuera del alcance de este trabajo y, por tanto, requiere de mayor investigación a modo de responderla adecuadamente.

\section{Referencias Bibliográficas}

Aguilar Rivera, José Antonio (1996). "El manto liberal: emergencias y constituciones". Política y Gobierno, vol. III, No 2, pp. 327-358.

Alenda, Stéphanie (2003). "Bolivia: la erosión del pacto democrático". Revista Fuerzas Armadas y Sociedad, vol. 18, $\mathrm{N}^{\mathrm{o}} 12,3-22$.

Buttolph Johnson, Janet, and Richard A. Joslyn (1995). Political Science Research Methods. Third Edition, Washington, D.C.: Congressional Quarterly.

Carbonell, Miguel (2008). "Neoconstitucionalismo y derechos fundamentales en tiempos de emergencia". Estudios Constitucionales, vol. 6, No 1, pp. 249-263.

Colomer Viadel, Antonio (1990). Introducción al constitucionalismo latinoamericano. Madrid: Ediciones de Cultura Hispánica. 
Cruz Villalón, Pedro (1984). Estados de excepción y suspensión de garantías. Madrid:Tecnos.

Delfino, María de los Ángeles (2000). "El desarrollo de los estados de excepción en las Constituciones de América Latina". En García de Enterría, Eduardo (compilador) Constitución y constitucionalismo hoy. Cincuentenario del Derecho constitucional comparado de Manuel García Pelayo. Caracas: Fundación Manuel García Pelayo.

Despouy, Leandro (1999). Los derechos humanos y los estados de excepción. México: UNAM.

Domingo, Pilar (2006). Bolivia. Fin de un ciclo y nuevas perspectivas políticas (1993-2003), Barcelona: Edicions Bellaterra.

Ferreira Rubio, Delia y Goretti, Matteo (1998). "WhenthePresidentGovernsAlone. The Decretazo in Argentina, 1989-1993". En Carey, John M. y Shugart,Matthew Soberg (eds) Executive DecreeAuthority, San Diego: Cambridge UniversityPress.

Figallo, Guillermo (1987). "Presentación", En: Diego García Sayán (ed.). Estados de emergencia en la región andina. Lima: Comisión Andina de Juristas.

Friedrich, Carl (1950). Constitutional Government and Democracy. Boston: Ginn and Company.

García Sayán, Diego (ed.) (1987). Estados de emergencia en la región andina. Lima: Comisión Andina de Juristas.

Gargarella, Roberto (2005). Los fundamentos legales de la desigualdad. El constitucionalismo en América (1776-1860). Madrid: Siglo XXI.

Glasser, Barney, y Anselm Strauss (1967). The Discovery of Grounded Theory. Strategies for Qualitative Research. California:Thousand Oaks, Sage Publications.

Gross, Oren, y Fionnuala Ni Aoláin (2006). Law in Times of Crisis: Emergency Powers in Theory and Practice. Cambridge: CUP.

Hagopian, Frances (2005). "Conclusions: Government Performance, Political Representation and Public Perceptions of Contemporary Democracy in Latin America", en: Frances Hagopian and Scott P. Mainwaring (eds), The Third Wave of Democratization in Latin America: Advances and Setbacks. New York: Cambridge University Press.

Iturralde, Manuel (2003). "Guerra y Derecho en Colombia: el decisionismo político y los estados de excepción como respuesta a la crisis de la democracia". Revista de Estudios Sociales, $N^{\circ} 15$, pp. $29-46$

Kruijt, Dirk, y Kees Koonings (2002). Las sociedades de miedo, Salamanca: Universidad de Salamanca.

Loveman, Brian (1993). The Constitution of Tyranny: Regimes of Exception in Spanish America, Pittsburgh: University of Pittsburgh. 
Loveman, Brian, y Davies, Thomas M. (1997). The Politics of Antipolitics. The Military in Latin America, Wilmington: Scholarly Resources Inc.

Maquiavelo, Nicolás (1987). Discursos sobre la primera década de Tito Livio, Madrid: Alianza.

Mesa, José, Teresa Gisbert, y Carlos Mesa Gisbert (2007). Historia de Bolivia. Sexta Edición Actualizada y aumentada, La Paz, Editorial Gisbert y Cia.

Neuendorf, Kimberly A. (2002). The Content Analysis Guidebook, London and New Delhi: Thousand Oaks, Sage Publications.

O’Donnell, Guillermo (1997). Contrapuntos: ensayos escogidos sobre autoritarismo y democratización, Buenos Aires: Paidós.

Ríos Álvarez, Lautaro (2009). "Defensa judicial de los derechos humanos en los estados de excepción". Estudios Constitucionales, vol. 7, Nº1, pp. 249-263.

Rossiter, Clinton (1946). Constitutional Dictatorship: Crisis Government in Modern Democracies, New Brunswick:Transaction.

Rouquié, Alain (1997). "Los militares en la política latinoamericana desde 1930". En Leslie Bethell (ed.) Historia de América Latina Política y Sociedad desde 1930, Barcelona: Crítica Grijalbo Mondadori.

Rousseau, Jean Jacques (1990). El contrato social, Madrid: Editorial Espasa Calpe.

Safford, Frank (1987). Politics, ideology and society, en: Leslie Bethell (ed.), Spanish America after Independence c. 1820-c.1870, New York: Cambridge University Press.

Vázquez Carrizosa, Alfredo (1979). El Poder Presidencial en Colombia: la Crisis Permanente del Derecho Constitucional. Bogotá: Enrique Dobry Editor.

Wilde, Marc de (2010). "Locke and the State of Exception. Towards a Modern Understanding of Emergency Government". European Constitutional Law Review, vol. 6, pp. 249-267.

Wright, Claire (2012). ¿De la seguridad nacional a la gestión de riesgos? El uso político de los regímenes de excepción en Bolivia, Ecuador y Perú, Ph.D. thesis, Universidad de Salamanca, España.

Wright, Claire (2012b). "Going Beyond the Roman Dictator: a Comprehensive Approach to Emergency Rule, with Evidence from Latin America". Democratization,Vol. 19, Nº4, pp. 713-734.

Zovatto, Daniel (1990). Los Estados de Excepción y los Derechos Humanos en América Latina, Caracas/ San José: Instituto Interamericano de Derechos Humanos/Editorial Jurídica Venezolana. 
